\title{
EVENT COLLISIONS IN SYSTEMS WITH DELAYED SWITCHES
}

\author{
J. Sieber ${ }^{* 1}$ P. Kowalczyk ${ }^{* *}$ \\ * Bristol Centre for Applied Nonlinear Mathematics, \\ Department of Engineering Mathematics, Queen's Building, \\ University of Bristol, BS8 1TR, U.K. \\ ** Institute of Mathematical Sciences, Laver Building, University \\ of Exeter, Exeter, EX4 4QE, U.K.
}

\begin{abstract}
We study dynamical systems that switch between two different vector fields depending on a discrete variable. If the two vector fields have linearly unstable fixed points and the switch is subject to a hysteresis and to a delay one expects the system to switch periodically back and forth between the two vector fields, always switching at certain submanifolds of the state space. This is true as long as the delay is sufficiently small. When the delay reaches a problem-dependent critical value so-called event collisions occur. We show that at these event collisions the switching manifolds can increase their dimension, giving rise to higher-dimensional dynamics near the periodic orbit than expected. In many practical applications such as control engineering the dynamical system has additional symmetry, which adds difficulty in the analysis because event collisions generically occur at several points along the periodic orbit simultaneously. Copyright $\odot 2006$ IFAC
\end{abstract}

Keywords: hybrid dynamical system, delayed switch, non-smooth map

\section{INTRODUCTION}

The motivation behind this paper is the observation that hybrid dynamical systems arising in practical applications often show a surprisingly intricate dynamical behavior if their switch is subject to a delay [Barton et al. (2005b); Bayer and an der Heyden (1998); Holmberg (1991)]. The observed behavior often does not match the simple classification of possible codimension-one bifurcations as given in [Sieber (2005)]. The reason behind this phenomenon is that practical systems often have special symmetries. For example piecewise affine systems as arising in control engineering have a full reflection symmetry. The presence of this symmetry causes a violation of the genericity assumptions made in [Sieber (2005)]. Most

\footnotetext{
1 The research of J.S. is supported by EPSRC grant GR/R72020/01.
}

prominently, periodic orbits of systems with delayed switching typically have 'corners'. A classical event collision corresponds to the case when, varying a parameter, one of the corners of the orbits crosses a switching boundary [Sieber (2005)]. In the examples of [Barton et al. (2005b); Bayer and an der Heyden (1998); Holmberg (1991)] the symmetry enforces that one of the other corners of the periodic orbit crosses a switching boundary simultaneously (at least for symmetric periodic orbits), which violates the assumptions of [Sieber (2005)]. This paper studys the simplest but most common case of symmetric hybrid systems, namely systems with full reflection symmetry. This symmetry is present in all examples studied in [Barton et al. (2005b); Bayer and an der Heyden (1998); Holmberg (1991)].

Let us consider hybrid dynamical systems of the form 


$$
\begin{aligned}
& \dot{x}(t)=f(x(t), u(t-\tau)) \\
& u(t)=\left\{\begin{aligned}
-1 \quad & \text { if } h(x) \geq 1, \text { or } \\
1 \quad & \text { if } h(x(t)) \in(-1,1) \text { and } u_{-}(t)=-1 \\
& \text { if } h(x(t)) \leq-1, \text { or, }
\end{aligned}\right. \\
&
\end{aligned}
$$

where $x(t) \in \mathbb{R}^{n}, u(t) \in\{-1,1\}, f: \mathbb{R}^{n} \times\{-1,1\} \mapsto$ $\mathbb{R}^{n}$ is smooth and $h: \mathbb{R}^{n} \mapsto \mathbb{R}$ is continuous and piecewise smooth with a nonzero gradient $H(x)=$ $\partial_{x} h(x)$. In the definition of $u(t)$ in $(1), u_{-}(t)$ is defined by

$$
u_{-}(t):=\lim _{s \nearrow t} u(s)
$$

which gives rise to a switch between $u=-1$ and $u=$ +1 with hysteresis between $h(x)=-1$ and $h(x)=1$. In control problems, typically, $x$ is the state variable, and $u$ is a discrete control input governed by the switching law in (1). For a trajectory $(x(\cdot), u(\cdot))$ of (1) we call $t$ a crossing time if $u(t) u_{-}(t)=-1$ (that is, $u(t) \neq u_{-}(t)$ ). The level sets $(-\infty,-1],(-1,1)$ and $[1, \infty)$ of the function $h$ provide a partition of $\mathbb{R}^{n}$ into disjoint sets with piecewise smooth boundaries given by $\{h(x)=$ $\pm 1\}$. Thus, at a crossing time $t, x(t)$ crosses either $\{h(x)=1\}$ from $\{h(x)<1\}$ to $\{h(x) \geq 1\}$, or $\{h(x)=$ $-1\}$ from $\{h(x)>-1\}$ to $\{h(x) \leq-1\}$. The state variable $x$ in system (1) follows at any instance of time one of the two flows $\varphi_{+}$or $\varphi_{-}$where $\varphi_{ \pm}$is generated by the ordinary differential equation $\dot{x}=f(x, \pm 1)$. That is, $x(t+\delta)=\varphi_{+}^{\delta} x(t)$ if $u([t-\tau, t-\tau+\delta])=1$, and $x(t+\delta)=\varphi_{-}^{\delta} x(t)$ if $u([t-\tau, t-\tau+\delta])=-1$.

A special feature of system (1) is that the switch introduced by $u$ is subject to a delay. That is, the evolution of $x$ depends, via $u$, on the value of $x$ from time $\tau$ ago. Typically, systems with delayed switches admit non-stationary, periodic or more complicated, dynamics [Barton et al. (2005a); Fridman et al. (2002); Holmberg (1991)]. The phase space of system (1) is infinite-dimensional because it is necessary to keep track of the history of $u$ in $[t-\tau, t]$ to determine the forward evolution of $x$ and $u$. More precisely, an appropriate initial value for (1) would be a tuple $\left(x_{0}, u_{0}\right)$ consisting of $x_{0} \in \mathbb{R}^{n}$, and a function $u_{0}$ : $\left[-\tau_{\max }, 0\right) \mapsto\{-1,1\}$ of, say, bounded variation (that is, $u \in B V:=B V\left(\left[-\tau_{\max }, 0\right] ;\{-1,1\}\right)$ where $\tau_{\max }$ is an upper bound for the delay). However, it is well known that the dynamics close to a periodic orbit $L=$ $(x(\cdot), u(\cdot))$ of (1) can be described locally by a finitedimensional smooth local return (Poincaré) map $M$ if $L$ satisfies the following two genericity conditions.

Condition 1. (Generic periodic orbits) All crossing times $t$ of $L$ satisfy the following two conditions.

(1) (Absence of event collision) The time $t-\tau$ is a not a crossing time of $L$.

(2) (Transversality) The gradient $H$ of $h$ is well defined and continuous in $x(t)$ and satisfies $H(x(t)) \dot{x}(t) \neq 0$.
Condition 1.1 automatically guarantees that $x(\cdot)$ is continuously differentiable in all crossing times, making the time derivative of $x$ in Condition 1.2 well defined. If the orbit $L$ is also slowly oscillating, that is, if the time differences between subsequent crossing times are all larger than the delay $\tau$, then the local return map $M$ is $n$-1-dimensional. In this case the map $M$ can be obtained by recording the first return map (Poincaré map) to a local cross-section (Poincaré section) in $\mathbb{R}^{n}$, transversal to the graph of $x(\cdot)$ in a point $x(t)$ where $u$ is constant in $[t-\tau, t]$. A reduction of the description of the dynamics of (1) near $L$ to the smooth finite-dimensional map $M$ links the bifurcation theory of slowly oscillating periodic orbits satisfying Condition 1 to the classical bifurcation theory of smooth finite-dimensional maps [Kuznetsov (2004)]. [Sieber (2005)] proves that this reduction to (higher-dimensional) smooth maps works also for periodic orbits that are not slowly oscillating as long as Condition 1 is satisfied. Furthermore, [Sieber (2005)] classifies what can happen generically near slowly oscillating periodic orbits that violate one of the conditions 1.1 or 1.2. It derives that the local return maps are piecewise smooth $n-1$-dimensional maps assuming certain secondary genericity conditions. This links the theory of codimension-one discontinuity-induced bifurcations of slowly oscillating periodic orbits to the theory of finite-dimensional piecewise smooth maps [Banerjee and Grebogi (1999); di Bernardo et al. (1999); Nordmark (1997); Nusse et al. (1994)].

The motivation behind this paper is the observation that many systems arising in applications have special symmetry properties that obstruct the application of the generic theory outlined in [Sieber (2005)]. The symmetry of the periodic orbit often implies that a collision (that is, the violation of Condition 1.1) for one crossing time $t$ leads automatically to a simultaneous collision for all other crossing times, which violates the secondary conditions assumed in [Sieber (2005)]. This has been observed in the example system studied extensively in [Barton et al. (2005b); Bayer and an der Heyden (1998)] as well as in many of the examples discussed in [Holmberg (1991)]. The major source of examples of systems of the form (1) is control engineering where often $f$ is linear and the switching law is affine, that is,

$$
\begin{aligned}
f(x, u) & =A x+b u \\
h(x) & =h^{T} x
\end{aligned}
$$

where $A \in \mathbb{R}^{n \times n}$, and $b, h \in \mathbb{R}^{n}$. The form (2) implies that system (1) has the $\mathbb{Z}_{2}$ symmetry of full reflection at the origin $(x, u) \mapsto(-x,-u)$, which occurs if the right-hand-side of (1) satisfies

$$
\begin{aligned}
f(x, u) & =-f(-x,-u) \\
h(x) & =-h(-x) .
\end{aligned}
$$

The $\mathbb{Z}_{2}$ reflection symmetry (3) typically gives rise to a symmetric periodic orbit $L=(x(\cdot), u(\cdot))$ satisfying $x(t-T)=-x(t)$ and $u(t-T)=-u(t)$ for the half- 
period $T$ and all $t$. An event collision of this symmetric periodic orbit for a crossing time $t$ automatically induces a simultaneous event collision for the crossing time $t-T$, a scenario which is not covered by the classification of [Sieber (2005)]. We point out that affine systems of the form (1), (2) can exhibit complex behavior, including chaos, even though all ingredients of the right-hand-side are linear. The switch governing $u$ in (1) is a strong nonlinearity which is a common cause of complicated dynamics.

\section{LOCAL RETURN MAPS OF SYMMETRIC PERIODIC ORBITS AT EVENT COLLISIONS}

Let us suppose that system (1), (3) has a symmetric periodic orbit $L_{*}=\left(x_{*}(\cdot), u_{*}(\cdot)\right)$ of half-period $T$ which, for a critical delay $\tau_{*}$, experiences an event collision for crossing time 0 , and, enforced by the reflection symmetry, for crossing time $T$. For compactness of presentation let us assume that 0 and $T$ are the only crossing times of $L_{*}$. Thus, $T=\tau_{*}$, and $x_{*}$ switches between the flows $\varphi_{+}$and $\varphi_{-}$at the crossing times 0 and $\tau_{*}$. Consequently, (without loss of generality) $L_{*}$ consists of the two segments

$$
\begin{aligned}
x_{*}\left(\left[0, \tau_{*}\right]\right) & =\varphi_{+}^{\left[0, \tau_{*}\right]}\left(x_{*}(0)\right), \\
u_{*}\left(\left[0, \tau_{*}\right)\right) & =-1, \text { and } \\
x_{*}\left(\left[\tau_{*}, 2 \tau_{*}\right]\right) & =\varphi_{-}^{\left[0, \tau_{*}\right]}\left(-x_{*}(0)\right)=-x_{*}\left(\left[0, \tau_{*}\right]\right), \\
u_{*}\left(\left[\tau_{*}, 2 \tau_{*}\right)\right) & =1 .
\end{aligned}
$$

Moreover, $h\left(x_{*}(0)\right)=1$ and $h\left(x_{*}\left(\tau_{*}\right)\right)=-1$. The following transversality condition guarantees that the evolution of system (1), (3) is continuous in $L_{*}$ :

Condition 2. (Continuous event collision of symmetric orbits) The orbit $L_{*}$ intersects the switching manifold $\{x: h(x)=1\}$ transversally at time 0 :

$$
q:=H\left(x_{*}(0)\right) f_{+} \cdot H\left(x_{*}(0)\right) f_{-}>0
$$

where $f_{+}=f\left(x_{*}(0), 1\right)$ and $f_{-}=f\left(x_{*}(0),-1\right)$.

Condition 2 means that, even though the orbit $x_{*}(\cdot)$ is not differentiable in its crossing times 0 and $\tau_{*}$, it still crosses the switching manifolds $\{x: h(x)= \pm 1\}$ transversally in the sense that the left- and the rightsided time derivatives of $x_{*}(\cdot)$ both point through the switching manifold and both point in the same direction. Condition 2 is formulated for crossing time 0 . The reflection symmetry implies that the same condition automatically holds also for the crossing time $\tau_{*}$. The point $p_{*}=\left(x_{*}(0), u_{*}\right)$ where

$$
u\left(\left[-\tau_{*}, 0\right)\right)=1, \quad u([-\tau-\Delta,-\tau))=-1
$$

is an element of $L_{*}$ (choosing $\Delta>0$ such that $\tau+$ $\Delta \leq \tau_{\max }$ for all $\tau \approx \tau_{*}$ ). Consider the following set of initial conditions in the vicinity of $p_{*}$ (denoting a sufficiently small neighborhood of a point $x \in \mathbb{R}^{n}$ by $U(x))$ :

$$
\begin{gathered}
U_{\tau}:=\left\{(x, u(\cdot)) \in \mathbb{R}^{n} \times B V: u((-\tau-\Delta,-\tau))=-1,\right. \\
\left.u([-\tau,-\tau+\Delta))=1, x \in U\left(x_{*}(0)\right)\right\} .
\end{gathered}
$$

The set $U_{\tau}$ is the set of initial conditions that switch exactly once from $\varphi_{-}$to $\varphi_{+}$in $U\left(x_{*}(0)\right)$. The point $p_{*}$ on the periodic orbit $L_{*}$ is an element of $U_{\tau_{*}}$. For a given delay $\tau$ the set $U_{\tau}$ can be identified with $U\left(x_{*}(0)\right)$, thus, defining a topology on $U_{\tau}$. Condition 2 guarantees that for delays $\tau$ close to $\tau_{*}$ the set $U_{\tau}$ is invariant relative to a sufficiently small neighborhood $\tilde{U}$ of $L_{*}$ in the following sense. The $x$-components of all trajectories starting from elements of $U_{\tau}$ switch exactly once from $\varphi_{-}$to $\varphi_{+}$in $U\left(x_{*}(0)\right)$, then follow $\varphi_{+}$to $U\left(x_{*}\left(\tau_{*}\right)\right)=U\left(-x_{*}(0)\right)$, then switch from $\varphi_{+}$ to $\varphi_{-}$in $U\left(x_{*}\left(\tau_{*}\right)\right)$, and then return to $U\left(x_{*}(0)\right)$, following $\varphi_{-}$(as long as the trajectory does not leave $\tilde{U}$ ). Consequently, for $\tau=\tau_{*}+\delta \approx \tau_{*}$ the system (1), (3) restricted to $U_{\tau}$ defines a return map $M_{\infty}: D\left(M_{\infty}\right) \subset$ $U_{\tau} \mapsto U_{\tau}$ where $D\left(M_{\infty}\right)$, the domain of definition of $M_{\infty}$, is an open subset of $U_{\tau}$. The identification of $U_{\tau}$ with $U\left(x_{*}(0)\right)$ identifies $M_{\infty}$ with a $n$-dimensional map $M$ from $U\left(x_{*}(0)\right)$ to $U\left(x_{*}(0)\right)$. The following theorem states that the $n$-dimensional map $M: U\left(x_{*}(0)\right) \mapsto$ $U\left(x_{*}(0)\right)$ describes the long-time behavior of system (1), (3) for small $\delta$ and near $L_{*}$ completely. Moreover, it provides a formula for the piecewise smooth $\operatorname{map} M$.

Theorem 3. (Dynamics near symmetric collisions) Let $\tau$ be sufficiently close to $\tau_{*}$ and let $\tilde{U}$ be a sufficiently small neighborhood of $L_{*} \subset \mathbb{R}^{n} \times B V$. Then all initial conditions in $\tilde{U}$ are mapped into $D\left(M_{\infty}\right)$ by system (1), (3) within a finite time less than $3 \tau_{*}$. On $D\left(M_{\infty}\right)$ the reduced map $M$ of $M_{\infty}$ is given as $M=F_{\tau}$ 。 $F_{\tau}$ where $F \tau: U\left(x_{*}(0)\right) \mapsto U\left(x_{*}(0)\right)$ is defined as

$$
F_{\tau}(x)=-\varphi_{+}^{\tau+t(x)} x
$$

and $t(x) \in(-\tau, \tau)$ is the unique time such that

$$
\begin{cases}h\left(\varphi_{+}^{t(x)} x\right)=1 & \text { if } h(x) \leq 1 \\ h\left(\varphi_{-}^{t(x)} x\right)=1 & \text { if } h(x)>1\end{cases}
$$

The definition of the traveling time $t(x)$ implies that $F_{\tau}$ is continuous in $D(M)$ and smooth in its two subdomains $D_{-}:=U\left(x_{*}(0)\right) \cap\{x: h(x) \leq 1\}$ and $D_{+}:=$ $U\left(x_{*}(0)\right) \cap\{x: h(x)>1\}$ but, in general, its derivative has a discontinuity along the boundary $D_{0}$ between $D_{-}$and $D_{+}$. The existence and uniqueness of the traveling time is a consequence of the transversality Condition 2. The linearizations of both parts of $F_{\tau}$ with respect to $x$ and $\tau$ in $x_{*}(0)$ and $\tau_{*}$ are:

$$
\begin{aligned}
& F_{\tau_{*}+\delta}\left(x_{*}(0)+\xi\right)-x_{*}(0)= \\
& \quad-A^{\tau_{*}}\left[\left[I-\frac{f_{+} H_{*}}{g}\right] \xi+\delta f_{+}\right]+O\left(\left|(\xi, \delta)^{2}\right|\right)
\end{aligned}
$$

where $H_{*}=H\left(x_{*}(0)\right), A^{\tau_{*}}=\partial_{x}\left[\varphi_{+}^{\left[0, \tau_{*}\right]} x\right]_{x=x_{*}(0)}, f_{+}=$ $f\left(x_{*}(0), 1\right)$, and 


$$
g=\left\{\begin{array}{cl}
H_{*} f_{+} & \text {if } x_{*}(0)+\xi \in D_{-} \backslash D_{0}, \\
-H_{*} A^{\tau_{*}} f_{+} & \text {if } x_{*}(0)+\xi \in D_{+} .
\end{array}\right.
$$

Condition 2 asserts that the product $q$ of $H_{*} f_{+}$and $H_{*} f_{-}=-H_{*} A^{\tau_{*}} f_{+}$is nonzero. Thus, Condition 2 implies that $g$ in nonzero in both cases. The affine approximation of the boundary $D_{0}$ between $D_{-}$and $D_{+}$in $x_{*}(0)$ is given by $\left\{x_{*}(0)+\xi: H_{*} \xi=0\right\}$. The map $F_{\tau}$ projects the whole subdomain $D_{-}$onto the $n$-1-dimensional local submanifold $\left\{x \in U\left(x_{*}(0)\right)\right.$ : $\left.h\left(-\varphi_{+}^{-\tau} x\right)=1\right\}$ which is the delayed switching manifold. Correspondingly, its linearization (6) projects $\xi$ linearly by $I-f_{+} H_{*} /\left(H_{*} f_{+}\right)$before propagating it by $-A^{\tau_{*}}$. Consequently, an event collision for a symmetric periodic orbit satisfying Condition 2 increases the dimension of the image of the local return map from $n-1$ in $D_{-}$to $n$ in $D_{+}$.

\section{ILLUSTRATIVE EXAMPLE}

This section will illustrate the most common scenario for the dynamics near an event collision of a symmetric periodic orbit. We will use a two-dimensional example of the piecewise linear form (1), (2) to demonstrate how the increase of the dimension of the return map of the periodic orbit manifests itself near an event collision.

We choose $h^{T}=(1,0)$ (without loss of generality) and the right-hand-side parameters $A$ and $b$ in (2) such that, for a critical delay $\tau=\tau_{*}$ system (1)-(2) has a symmetric periodic orbit $L_{*}=\left(x_{*}(\cdot), u_{*}(\cdot)\right)$ with halfperiod $\tau_{*}$ and crossing times 0 and $\tau_{*}$. Furthermore, we choose $A, b$ and $\tau_{*}$ in a manner such that $A, b$ and $L_{*}$ satisfy the following conditions.

(a) The matrix $A$ and the critical delay $\tau_{*}$ satisfy $A^{\tau_{*}}:=\exp \left(A \tau_{*}\right)=r\left[\begin{array}{rr}0 & -1 \\ 1 & 0\end{array}\right]$ with $r>1$.

(b) The colliding orbit $x_{*}$ intersects the switching line $\left\{x: h^{T} x=1\right\}$ in $x_{*}(0)=\left(1, z_{*}\right)^{T}$ and the switching line $\left\{x: h^{T} x=-1\right\}$ in $x_{*}\left(\tau_{*}\right)=$ $\left(-1,-z_{*}\right)^{T}$ transversally. That is, $h^{T} f_{+}>1$ and $h^{T} f_{-}>0$ where

$$
\begin{aligned}
f_{+} & :=\left.\frac{d}{d t} \varphi_{+}^{t}\right|_{t=0} x_{*}(0)=A x_{*}(0)+b \\
f_{-} & :=\left.\frac{d}{d t} \varphi_{-}^{t}\right|_{t=0} x_{*}(0)=A x_{*}(0)-b=-A^{\tau_{*}} f_{+} .
\end{aligned}
$$

The coordinate $z_{*}$ is uniquely defined by $A$ and $\tau_{*}$ (chosen as required in point (a)) and $f_{+}$. The concrete expression for $z_{*}$ is given in the Appendix A.

(c) The vector $f_{+}$has the form $f_{+}=(1, c)^{T}$, which implies that $f_{-}=(r c,-1)$ due to point (a), and $c>0$ due to point (b).

Thus, both crossing times of $L_{*}$ violate genericity condition 1.1 simultaneously. Figure 1(a) shows a phase portrait of $L_{*}$ (grey, thick, solid curve), superimposed with the non-delayed switching lines $\left\{h^{T} x=\right.$
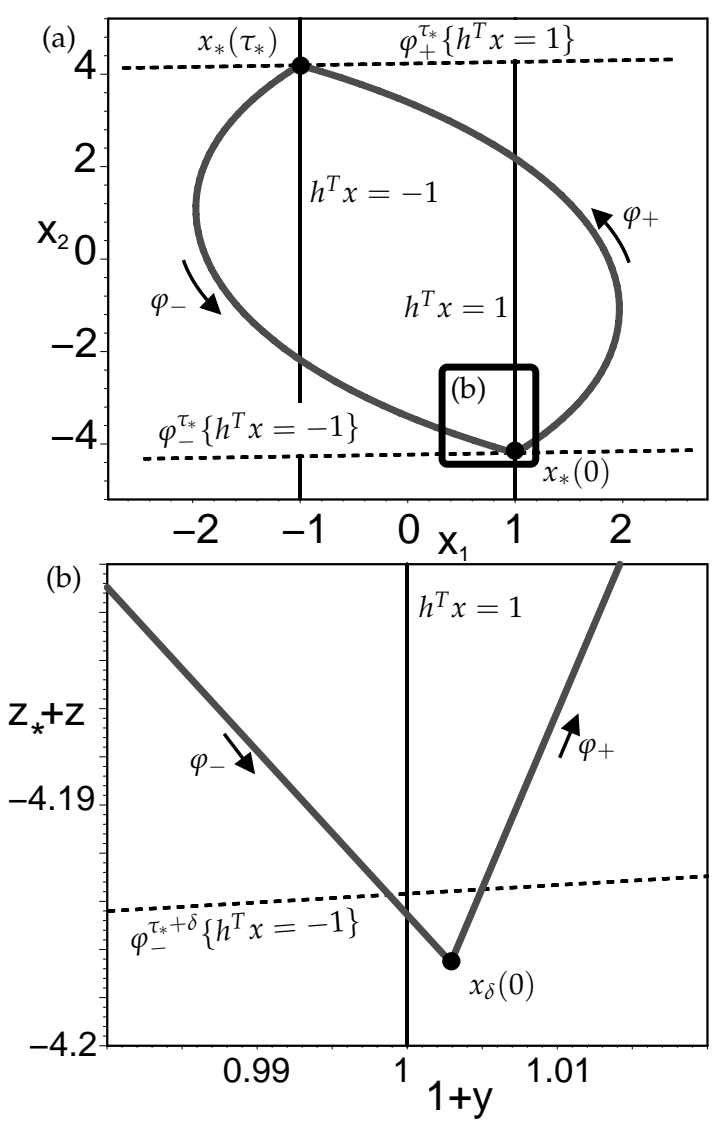

Fig. 1. Phase portrait of a colliding periodic orbit as discussed in Section 3. The dashed lines are the time $\tau_{*}$ images of the lines $\left\{h^{T} x=y= \pm 1\right\}$ under the flows $\varphi_{+}$and $\varphi_{-}$, respectively. The periodic orbit $L_{*}$ (in (a)) switches at times 0 and $\tau_{*}$ due to the crossing of the line $\{y= \pm 1\}$ time $\tau_{*}$ ago simultaneous to its crossing of the other switching line $\{y=\mp 1\}$. How this collision is unfolded by increasing the delay to $\tau_{*}+\delta$ is shown in the zoom (b). The delay $\tau_{*}+\delta$ is larger than the half-period and the periodic orbit no longer switches at the delayed switching lines.

$\pm 1\}$ (solid) and their time $\tau_{*}$ images $\varphi_{ \pm}^{\tau_{*}}\left\{h^{T} x= \pm 1\right\}$ (dashed).

Condition (a) means that $A$ has a complex pair of eigenvalues with positive real part and that $\tau_{*}$ is such that the rotation angle $\alpha$ induced by $\exp \left(A \tau_{*}\right)$ is $\pi / 2$ (which results in the simple form of $\exp \left(A \tau_{*}\right)$ in point (a)). Condition (b) corresponds to the transversality Condition 2 of Section 2. If condition (b) is satisfied the choice $f_{+}=(1, c)$ can be made without loss of generality. Appendix A describes in detail how the parameters $r, c$ and $\alpha$ uniquely define $A, b$, the critical delay $\tau_{*}$, and the colliding symmetric periodic orbit $x_{*}(\cdot)$ of period $2 \tau_{*}$. The requirement that the delay $\tau_{*}$ is non-negative and the condition $h^{T} f_{-}>0$ (part of condition (b)) restrict the set of admissible $r$ and $c$ by the conditions $r>1, c>0$, and

$$
c>\frac{r \pi-2 \log r}{\pi+2 r \log r}
$$


(see Appendix A).

The linearizations (6) and (7) (where $H_{*}=(1,0)$ ) approximate the half map $F_{\tau}$ of the local return map (which is nonlinear, even though system (1),(2) is piecewise affine) for $x=\left[1, z_{*}\right]^{T}+[y, z]^{T}$ and $\tau=\tau_{*}+$ $\delta$ for small $[y, z]^{T}$ and $\delta$. Expressed in the quantities $r, c,[y, z]$ and $\delta$ the linearized $F_{\tau}$ reads (calling the truncation to affine terms by $F_{\text {lin }}$ )

$$
F_{\text {lin }}:\left[\begin{array}{l}
y \\
z
\end{array}\right] \mapsto\left\{\begin{array}{cc}
{\left[\begin{array}{c}
-r c y+r z+r c \delta \\
-r \delta \\
-y+r z+r c \delta \\
\left(c^{-1}-r\right) y-r \delta
\end{array}\right]} & \text { if } y \leq 0
\end{array}\right.
$$

Hence, if $c>r$ (then also restriction (8) is satisfied) the map $F_{\text {lin }}$ has the fixed point

$$
\left[\begin{array}{l}
y_{0} \\
z_{0}
\end{array}\right]=\left\{\begin{array}{cc}
{\left[\begin{array}{c}
r \delta(c-r) /(r c+1) \\
-r \delta
\end{array}\right]} & \text { if } \delta<0, \\
{\left[\begin{array}{c}
r \delta c(c-r) /\left(2 c+r^{2} c-r\right) \\
-r \delta c(r c+1) /\left(2 c+r^{2} c-r\right)
\end{array}\right]} & \text { if } \delta>0,
\end{array}\right.
$$

Thus, there exists a continuous family $x_{\delta}=\left[y_{\delta}, z_{\delta}\right]^{T}$ of fixed points of $F_{\text {lin }}$ for $\delta \in\left(-\delta_{0}, \delta_{0}\right)$. This continuous family persists under the small perturbation toward $F_{\tau}$. The fixed points of $F_{\tau}$ correspond to a family of symmetric periodic orbits of system (1), (2) which has an event collision at $\tau=\tau_{*}$ (i.e., $\delta=0$ ). The fixed point $x_{\delta}$ has only one nonzero eigenvalue (corresponding to a nonzero Floquet multiplier of the periodic orbit) for $\delta<0$. This eigenvalue is $r c>1$. Hence, $x_{\delta}$ is unstable with only one nontrivial direction.

When $\delta>0$, the fixed point $x_{\delta}$ has a complex conjugate pair of eigenvalues $-1 / 2 \pm \sqrt{1 / 4-r^{2}+r / c}$ if $c>(r-1 /(4 r))^{-1}$ which is stable if $c<(r-1 / r)^{-1}$, loosing its stability in a $1: 3$ resonant torus bifurcation at $c=(r-1 / r)^{-1}$. Consequently, for $r \in(\sqrt{5} / 2, \sqrt{2})$ and for all $c \in\left(r,(r-1 / r)^{-1}\right)$ there exists a stable symmetric periodic orbit of system (1), (2) with a stable complex conjugate pair of Floquet multipliers for all $\tau=\tau_{*}+\delta$ and $\delta>0$ sufficiently small. The degeneracy of the strong (1:3) resonance is caused by our selection of the rotation angle $\alpha=\pi / 2$ in the choice of the parameters in $A$ and $\tau_{*}$. In general, no torus will emanate from this resonant torus bifurcation [Kuznetsov (2004)]. Variation of the angle $\alpha$ unfolds this degeneracy.

Figure 2 shows the iterations of the nonlinear map $F_{\tau}$ (the exact negative half-return map of the system (1), (2)) for $\tau=\tau_{*}+\delta$ where $\delta=0.1, c=3 / 2$ and $r=(1+\sqrt{10}) / 3 \approx 1.39$ (Fig. 2(a)) and $r=\sqrt{2}$ (Fig. 2(b)). Fig. 2(a) gives evidence of a stable fixed point with two complex conjugate eigenvalues in approximate 1:3 resonance whereas Fig. 2(b) clearly shows an unstable fixed point at the center (and, possibly, the period three saddle-type orbit which is generically present near $1: 3$ resonances). Fig. 1(b) shows a zoom-in into the neighborhood of $x_{*}(0)$ of the phase
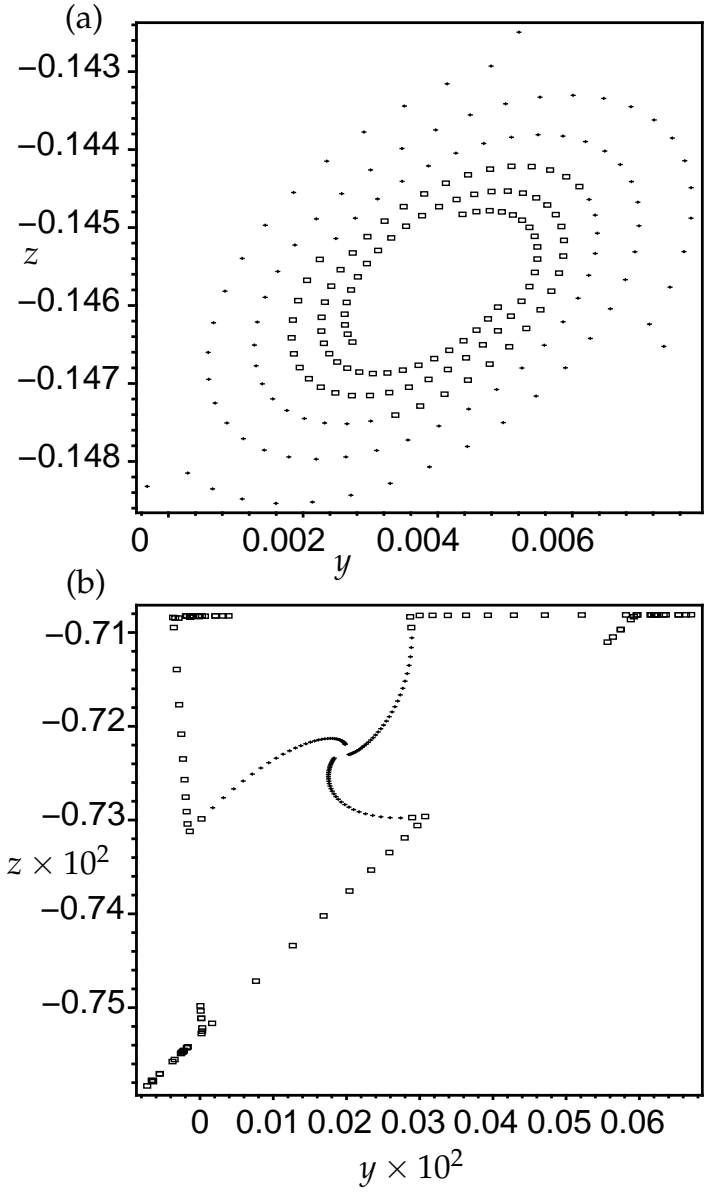

Fig. 2. Iterations of the half-return map $F_{\tau}$ starting near the fixed point. The first 100 iterates are marked by crosses, the iterates 101-200 are marked by boxes, giving evidence of a stable fixed point for $c=3 / 2, r=(1+\sqrt{10}) / 3 \approx 1.39, \delta=0.1(\tau=$ $\tau_{*}+\delta$ ) and an unstable fixed point for $c=3 / 2$, $r=\sqrt{2}$.

portrait of the periodic orbit $L_{\delta}$ where $\delta=0.1$ corresponding to the stable fixed point in Fig. 2(a). The periodic orbit $L_{\delta}$ does not switch at the delayed switching line $\varphi_{-}^{\tau_{*}+\delta}\left\{h^{T} x=-1\right\}$. The half-period of $L_{\delta}$ is less than $\tau_{*}-\delta$. Thus, $L_{\delta}$ is a stable symmetric periodic orbit that is not slowly but rapidly oscillating. Hence, this example also illustrates that a stable symmetric periodic orbit can be created in an event collision by increasing the delay beyond the critical value $\tau_{*}$.

\section{CONCLUSION}

The paper discusses the dynamics near periodic orbits in hybrid dynamical systems. It is motivated by the fact that in many practical applications the presence of symmetry prevents the generic and simple bifurcation scenarios as classified in [Sieber (2005)] but instead gives rise to intricate and counterintuitive event collision phenomena [Holmberg (1991); Barton et al. (2005b); Bayer and an der Heyden (1998)]. 
We describe and unfold the simplest and most common case of an event collision in a symmetric system as it occurs, for example, in a piecewise affine system which is switching with hysteresis and delay. In this case two corners of a symmetric periodic orbit simultaneously collide with a switching manifold. This causes an increase of the dimension of the phase space for the return map along the periodic orbit. We demonstrated this fact with a simple two-dimensional piecewise linear example which has a symmetric periodic orbit with a weakly stable or unstable complex conjugate pair of eigenvalues if the delay is greater than the critical value corresponding to an event collision.

The analysis of the possible dynamics in the unfolding of the event collision is far from complete but the initial theoretical results presented in this paper will lead to classifications of practically relevant behavior for concrete systems.

\section{REFERENCES}

Banerjee, S., and C. Grebogi. Border collision bifurcations in twodimensional piecewise smooth maps. Phys. Rev. E, 59:4052-4061, 1999.

Barton, D.A.W., B. Krauskopf, and R.E. Wilson. Explicit periodic solutions in a model of a relay controller with delay and forcing. Nonlinearity, 18(6): 2637-2656, 2005a.

Barton, D.A.W., B. Krauskopf, and R.E. Wilson. Periodic solutions and their bifurcations in a nonsmooth second-order delay differential equation. Preprint 2005.15, University of Bristol, Bristol Centre for Applied Nonlinear Mathematics, 2005b. http://www.enm.bris.ac.uk/anm/preprints /2005r15.html. (to appear in: Dynamical Systems: An International Journal)

Bayer, W., and U. an der Heyden. Oscillation types and bifurcations of a nonlinear second-order differential-difference equation. J. Dynam. Diff. Eq., 10(2):303-326, 1998.

di Bernardo, M., M.I. Feigin, S.J. Hogan, and M.E. Homer. Local analysis of C-bifurcations in $\mathrm{n}$ dimensional piecewise smooth dynamical systems. Chaos, Solitons and Fractals, 10:1881-1908, 1999.

Fridman, L., E. Fridman, and E. Shustin. Steady modes and sliding modes in relay control systems with delay. In J. P. Barbot and W. Perruguetti, editors, Sliding Mode Control in Engineering, pages 264-295. New York, 2002.

Holmberg, U. Relay feedback of simple systems. PhD thesis, Lund Institute of Technology, 1991.

Kuznetsov, Y.A. Elements of Applied Bifurcation Theory. Springer Verlag, 2004. third edition.

Nordmark, A.B. Universal limit mapping in grazing bifurcations. Phys. Rev. E, 55:266-270, 1997.

Nusse, H., E. Ott, and J. Yorke. Border collision bifurcations: an explanation for observed bifurcation phenomena. Phys. Rev. E, 49:1073-1076, 1994.
Sieber, J. Dynamics of delayed relay systems. Preprint 2005.18, University of Bristol, Bristol Centre for Applied Nonlinear Mathematics, 2005. http: //www.enm.bris.ac.uk/anm/preprints /2005r18.html.

\section{Appendix A. RECONSTRUCTION OF THE RIGHT-HAND-SIDE}

This appendix describes in which sense the righthand-side parameters $A, b$ and $\tau_{*}$ of the piecewise affine system (1),(2) are uniquely determined by the artificial parameters $c, r$ and $\alpha$ which we use in Section 3 to describe the return map of the colliding periodic orbit $L_{*}$.

Let us denote the first component of the state variable $x \in \mathbb{R}^{2}$ by $y$ and the second component by $z$. We choose $h=(1,0)^{T}$ and the matrix $A=\tau_{*}^{-1} A_{0}$ where

$$
A_{0}=\left[\begin{array}{cc}
\log r & -\alpha \\
\alpha & \log r
\end{array}\right]
$$

and $r>1$. Thus, the flows $\varphi_{ \pm}$are given by

$$
\varphi_{ \pm}^{t} x=\exp (A t) x \pm(\exp (A t)-I) \tau_{*} A_{0}^{-1} b,
$$

spiraling outward from the unstable sources $\mp \tau_{*} A_{0}^{-1} b$. Consequently, system (1),(2) with delay $\tau_{*}$ has a symmetric periodic orbit that switches from $\varphi_{-}$to $\varphi_{+}$ in $x_{*}(0)=\left(1, z_{*}\right)^{T}$ on the switching line $h^{T} x=1$ if

$$
-\left[\begin{array}{c}
1 \\
z_{*}
\end{array}\right]=\exp A_{0}\left[\begin{array}{c}
1 \\
z_{*}
\end{array}\right]+\left(\exp A_{0}-I\right) \tau_{*} A_{0}^{-1} b .
$$

Furthermore, if we prescribe the time derivative of the outgoing flow $\varphi_{+}$in $\left(1, z_{*}\right)^{T}$ by $(1, c)$ then the parameters $A, b$ and $\tau_{*}$ and the periodic orbit are uniquely determined by the parameters $r, \alpha$ and $c$ and the relations

$$
\begin{aligned}
{\left[\begin{array}{c}
\tau_{*}^{-1} \\
\tau_{*}^{-1} z_{*}
\end{array}\right]=} & \frac{1}{2}\left[I-\exp A_{0}\right] A_{0}^{-1}\left[\begin{array}{l}
1 \\
c
\end{array}\right], \\
b \quad= & {\left[\exp A_{0}+I\right]\left[\begin{array}{l}
1 \\
c
\end{array}\right] } \\
x_{*}( \pm t)= & \exp \left( \pm A_{0} \tau_{*}^{-1} t\right)\left[\begin{array}{c}
1 \\
z_{*}
\end{array}\right] \pm \\
& {\left[\exp \left( \pm A_{0} \tau_{*}^{-1} t\right)-I\right] \tau_{*} A_{0}^{-1} b }
\end{aligned}
$$

where $A_{0}$ is given by (A.1) and $t$ runs from 0 to $\tau_{*}$. Hence, the relations (A.2) allow one to study the return map near the colliding periodic orbit in dependence of the parameters $r, c$ and $\alpha$. We choose $r>1$ and, for convenience, $\alpha=\pi / 2$ in Section 3, implying that $\exp A_{0}=\left[\begin{array}{cc}0 & -r \\ r & 0\end{array}\right]$. This results in the relation

$$
\tau_{*}=\frac{4 \log ^{2} r+\pi^{2}}{2 \log r-r \pi+c \pi+2 c r \log r} .
$$

for $\tau_{*}$. The condition $\tau_{*}>0$ implies the admissibility condition on $c$

$$
c>\frac{r \pi-2 \log r}{\pi+2 r \log r},
$$

which is always satisfied if $c>r>1$. 